\title{
Simulating Gaia observations and on-ground reconstruction
}

\author{
E. Masana, C. Fabricius, J. Torra, J. Portell and J. Catañeda \\ Departament d'Astronomia i Meteorologia. Universitat de Barcelona \\ Av. Diagonal 647, E 08028 Barcelona, Spain \\ email: emasana@am.ub.es
}

\begin{abstract}
On a daily basis the Gaia telemetry data (some 30 GB) must be stored and treated in order to reconstruct the actual observations. This initial data treatment processes all newly arrived telemetry and various pieces of auxiliary data. The first part of the process is merely a reformatting to create raw objects for permanent storage in the raw data base (some 40 $\mathrm{TB}$ at the end of the mission). The next part is to analyze the data to derive initial values for the observables, e.g. transit times and fluxes, producing intermediate objects. Finally, the intermediate objects are matched with sources in the data base, linking all the observations of a given source.

To check the initial data treatment algorithm we use simulations of the telemetry stream provided by GASS, the Gaia System Simulator. GASS simulates astrometric, photometric and radial velocity data, using models of the satellite and on-board instruments, as well as the models of different of objects observed by Gaia (stars, galaxies, solar system objects, ...). On the other hand, the initial data treatment allows us to validate the data generated by GASS, which are used too to check other algorithms like the First Look or the Astrometric Global Iterative Solution (AGIS).
\end{abstract}

Keywords. astrometry, methods: data analysis

\section{Introduction}

The daily Gaia telemetry stream is reformatted and stored in the raw data base as raw objects (about $40 \mathrm{~TB}$ at the end of mission). The observations are cross-matched and stored in the Main Data Base (MDB). Different algorithms, such as Astrometric Global Iterative Solution (AGIS), Photometric Global Iterative Solution (PGIS) or specific algorithms from the Coordination Units of the Data Processing and Analysis Consortium (DPAC), work with the objects stored in the MDB to achieve the final results.

\section{The Gaia System Simulator}

The Gaia System Simulator (GASS) is part of the Gaia Simulator, a set of three data generators designed to cover the simulation needs of the Gaia community. GASS simulates astrometric, photometric and radial velocity telemetry data, using models of the satellite and on-board instruments, as well as the models of objects observed by Gaia such as stars, galaxies, solar system objects, quasars.

\section{The Initial Data Treatment}

Initial Data Treatment (IDT) is a data processing system that receives raw telemetry data from the spacecraft (or from the Gaia System Simulator during the design phase) 
and outputs basic image parameters of the observations. IDT computes:

- Refined attitude data generated from raw attitude.

- Image parameter: transit times, centroids and fluxes.

- Raw astrometric, photometric and spectrometric data.

- Approximate celestial coordinates for source identification.

The data processing chain that forms the core of IDT includes also calculations such as the background of the measured objects and their colors.

\section{Validation of on-ground reconstruction}

An automatic validation tool has been developed to compare the results of IDT with the ideal values provided by GASS. It has demonstrated the feasibility of the on-ground sky reconstruction using real mission observations.

The first trial with a dataset with 10 millions of sources observed over 400 days shows a correct reconstruction of the celestial coordinates and photometry of the sources, as well as validates the high-precision observations, required for the Astronomical Iterative Solution.

\section{Acknowledgements}

This project is supported by the Spanish MEC under contract PNE2006-13855-C02-01.

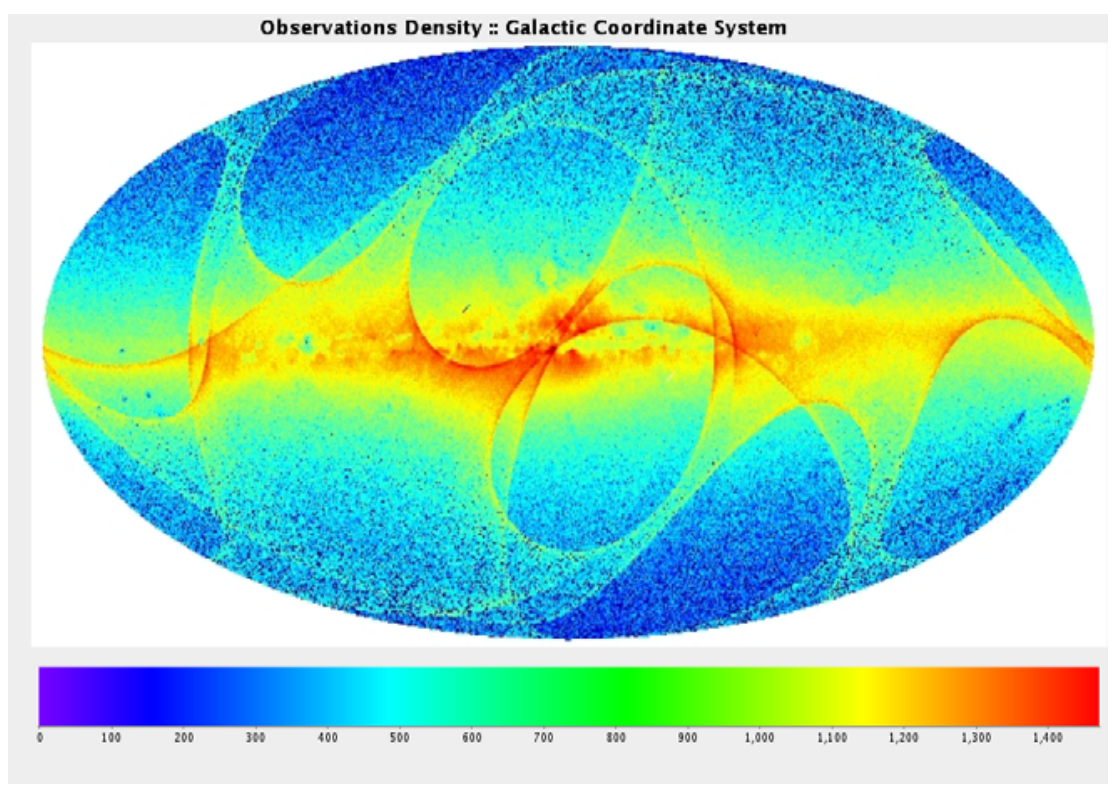

Figure 1. On-ground reconstruction of the sky: density of observations per square degree for a six months simulation. The observed pattern is a combination of the Galaxy Model and the Nominal Scanning Law of Gaia. 